\title{
„An dem Tag, als JHWH ihn rettete aus der Hand aller seiner Feinde und aus der Hand Sauls“ (Ps 18,1)
}

\author{
Erwägungen zur Anordnung der biographischen Angaben zu David im \\ Psalter
}

\begin{abstract}
Beat Weber
Theologisches Seminar Bienenberg, Liestal (Schweiz) \& Department of Ancient Languages, University of Pretoria, Pretoria (South Africa) weber-lehnherr@sunrise.ch
\end{abstract}

\begin{abstract}
Examining the arrangement of biographical notices in the titles of psalms ascribed to David reveals that these are not placed haphazardly. Their order and correlation conveys a message to the reader of the first two Davidic collections of psalms. There are two main horizons, one reflecting on the conflict between Saul and David (Pss 34; 52-59, followed by Ps 60 with national enemies), another reflecting on the conflict between David and Absalom (Ps 3-7; 63). The headings of Pss 18 (doubling 2 Sam 22) and Ps 51 (in connection with Ps 2 and 72 ) are of special hermeneutical significance, as they guide the reader's understanding of such biographical information, signalling relationships between the headings of different psalms als well as back to the narrative of the Book of Samuel (1-2 Sam). In the midst of prayers in which David is struggling with enemies, Ps 18 foregrounds the salvation of JHWH "at the end." Pss 2; 51 and 72 are interconnected by the common theme of the house of David and the prophetic voice of Nathan adressed twice to king David (2 Sam 7; 12).
\end{abstract}

\section{Keywords}

David ; Psalms ; $1 / 2$ Samuel ; titles ; 2 Sam 22 ; Ps 18 
In der neueren Psalmen- und Psalterforschung ist den Präskripten, die sich

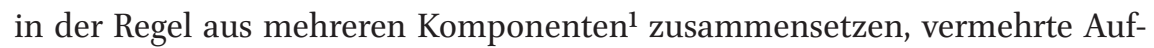
merksamkeit zuteil geworden. ${ }^{2}$ Es handelt sich bei ihnen um Texte sui generis, nämlich um Metatexte, die multiple Ein- und Zuordnungen markieren und aktivieren. ${ }^{3}$ Deren Verweissystem kann in einem Schema folgendermassen dargestellt werden:

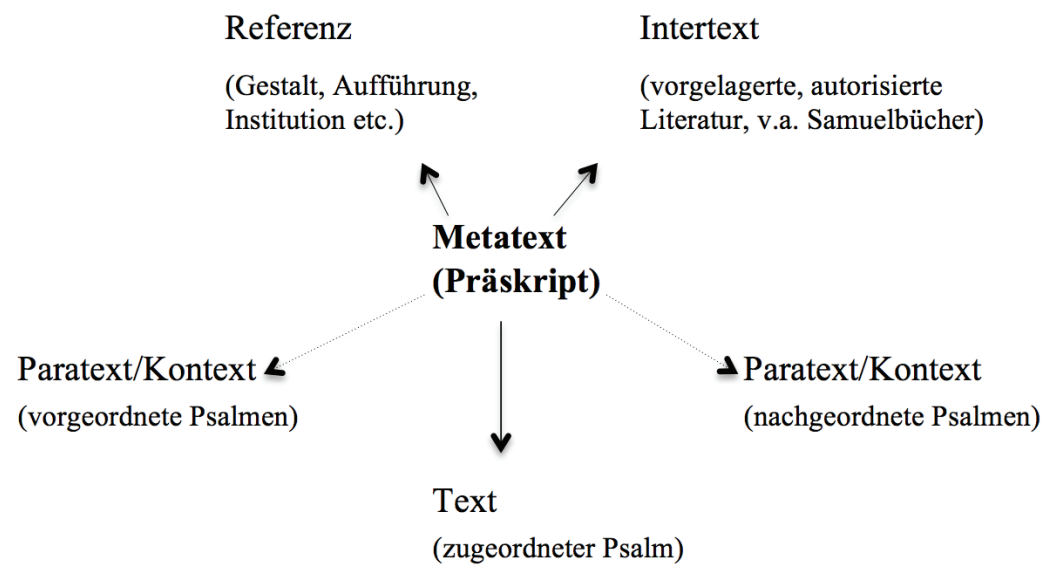

1 Es lassen sich unterschiedliche Komponenten („slots“) unterscheiden (vgl. Bayer, „Titles“, S. 47-73). Dazu gehören: Gattungsangaben, diverse Angaben zur Performanz (Melodie, Instrumentierung, liturgische und didaktische Hinweise), Zuschreibungen an Person(gruppen), Situationsangaben.

2 Vgl. zuletzt Janowski, „Hindin“.

3 Eine Reihe Faktoren trägt zur Komplexität dieses Verweissystems bei, zumal einzelne Angaben änigmatisch bleiben oder Aufführungshinweise nicht aufgehellt werden können. Die einzelnen Komponenten dürften zu unterschiedlichen Zeiten - auf dem (möglichen) Weg vom Einzelpsalm bis zur redaktionellen Endgestalt des Buches - beigegeben worden sein. Zudem kann jede Präskript-Komponente unterschiedliche Relationen gewichten, mit anderen in Interaktion treten und Bedeutungsmodifikationen (z.B. in Richtung einer Autorschaft der/aller David-Psalmen) erfahren. Schliesslich haben diesbezügliche Unterschiede in den Textüberlieferungen (мт, Handschriften aus den Höhlen am Toten Meer, LXX) Erklärungsbedarf (wir konzentrieren uns in dieser Studie auf den Befund іm мт). 
לדוד ist die häufigste Präskript-Komponente im MT (73mal). Von ihr her werden alle in der Skizze notierten Relationen aktiviert. Die mit dem Namen verbundene Präposition lamed markiert Zugehörigkeit. ${ }^{4}$ Die Wendung verweist auf eine - wie immer geartete - Beziehung zwischen Gestalt und Text und regt derart an, den nachfolgenden Psalm mit David in Verbindung zu bringen. Referenz und Intertextualität kommen insofern zum Zug, als auf eine geschichtliche Gestalt Israels abgestellt wird, die in bestimmten Texten greifbar ist. Schliesslich ergeben sich angesichts der Platzierung im Buch und damit der Kontextuierung mit anderen Psalmen und deren Präskripten Sinnzuschüsse (Assoziierung, konnektive und disjunktive Momente etc.). ${ }^{5}$

Psalmen mit David-Präskripten sind hauptsächlich in zwei grossen Gruppen gesammelt (Ps 2/3-41* und Ps 51-72*) und machen den Hauptbestand der Psalterteilbücher I (Ps 1-41) und II (Ps 42-72) aus. ${ }^{6}$ Bei einigen dieser Psalmen findet sich, stets nachgestellt, als ergänzende Komponente eine biographisch-situative Angabe zum Leben Davids. ${ }^{7}$ Es handelt sich um die folgenden dreizehn Psalmen: Ps $3 ; 7 ; 18 ; 34 ; 51 ; 52 ; 54 ; 56 ; 57 ; 59 ; 60 ; 63 ; 142 .{ }^{8}$ Sie setzen die David-Präskribierungen voraus und verstärken aufgrund ihrer Konkretisierung den David-Bezug. Anhand eines angetippten Hinweises wird dergestalt ein Lesemodus angesteuert, demzufolge der nachfolgende Psalm im Kontext von Geschehen und Geschichte, erzählt in den Samuelbüchern, zu

4 Die Präposition zeigt zunächst eine allgemeine, nicht näher bestimmte Relationalität an: „zugehörig David“ (Jenni, Präpositionen 3, S. 71, klassifiziert die Belege als „Lamed inscriptionis“). Je nach Kontext können sich Bedeutungsverschiebungen in Richtung einer Dedikation (z.B. לשלמה Ps 72,1, im Licht des Kolophons Ps 72,20) oder einer Autorangabe (z.B. in den Präskripten von Ps 7; 18) einstellen (vgl. Rösel, Redaktion, S. 161-163; Kleer, Sänger, S. 78-86). Letzteres hat sich im Verlauf der Deutungs- und Rezeptionsgeschichte für den Psalter insgesamt durchgesetzt (vgl. Weber, Werkbuch III, S. 165f.223-227.263f.).

5 Vgl. Wilson, Editing, S. 155-158.

6 Dazu kommt in Teilbuch III der in die zweite Qorach-Gruppe eingeschobene Ps 86 sowie Solitäre und Kleingruppen in den eine spätere Redaktionsstufe darstellenden Teilbüchern IV (Ps 101; 103) und V (Ps 108-110; 122; 124; 131; 133; 138-145).

7 Diese sind gegenüber der David-Zuschreibung entsprechend als gleich- oder (eher) nachzeitig einzustufen und werden - je nach Einschätzung - als historisch, historisierend oder midraschartig charakterisiert.

8 Ps 9 bleibt unberücksichtigt. Es gibt zwar Versuche, den rätselhaften Ausdruck עלמות לבן על על Präskript in diesem Sinn zu verstehen (על־מות לבן, על̈ber den Tod/wegen des Sterbens [vgl. Ps 48,15], in Bezug auf den Sohn“ o.ä.) und auf Absalom zu beziehen (vgl. Millard, Komposition, S. 133f.). Der Umstand, dass die situativ-biographischen Angaben (sonst) stets nach der David-Zuschreibung erscheinen, widerrät jedoch dieser Annahme. 
deuten ist. ${ }^{9}$ Dabei wird vorausgesetzt, dass die Lesenden, Meditierenden und (Nach-)Betenden von Psalm und Psalter diese Überlieferung kennen und sie mit dem poetisch verfassten Psalm in Verbindung zu bringen vermögen (memorierende Vergleichzeitigung). ${ }^{10}$ Dem Psalm kommt gegenüber der Geschichtserzählung dabei eine gebetstheologische Vertiefung und/oder belehrende Funktion zu.

Nach früheren Arbeiten ${ }^{11}$ haben sich in jüngerer Zeit Martin Kleer und Vivian Johnson monographisch mit den biographischen David-Präskripten befasst. ${ }^{12}$ Anhand obiger Skizze ausgedrückt: Johnson erarbeitet die vom Metatext (biographische Angaben) angezielten Verbindungen zwischen Text (Psalm) und Intertext (David- und weitere Erzählungen). In der stärker diachron orientierten und weiter gefassten Studie von Kleer geht es um Facetten und Entwicklungen des "Davidbildes“ in den Psalmen (sowie Sir und ${ }_{11 Q P s}{ }^{a}$ ). Die David-Angaben in den Präskripten werden als gestaffelt zugewachsen verstanden und die von ihnen ausgehende Bedeutung im Gegenüber von Texten und Intertexten profiliert. Anders als Johnson nimmt er die Buchredaktion in den Blick, wodurch die Para- bzw. Kontexte (Psalmenvorfeld und -nachfeld) einbezogen werden. Diese „Seitenbezüge“ innerhalb des Dreiecks: Metatext Text - Paratext/Kontext (s.o., Skizze) stehen im Fokus der vorliegenden Studie. Insofern knüpft sie an diejenige von Kleer an, hat aber (wie Johnson) einen synchronen Ansatz. Wir fragen: Wie sind die biograpischen David-Angaben (und die mit ihnen präskribierten Psalmen) ${ }^{13}$ untereinander verbunden? Welche eingelagerten Lesestrategien und Rezeptionsprozesse verbinden sich mit ihnen in der Fortlesung des Psalters? ${ }^{14}$ Mit der Fragestellung verbindet

Teilweise sind die metatextlichen Angaben mehrdeutig, so dass vermutlich mehrere Geschehnisse aus dem Leben Davids (und allenfalls darüber hinaus in anderen Erzähltexten) in Erinnerung gerufen werden (sollen).

10 Es handelt es sich dabei nicht um ein isoliertes Phänomen, vielmehr hat es Anteil an der im Psalter eingeschriebenen Sekundarität im Sinne einer Rückbezüglichkeit auf vorgelagertes Schrifttum (vgl. Weber, „Psaltergenese“, S. 737). Die Doppelüberlieferung 2 Sam 22 $\approx$ Ps 18, die Programmatik des Eröffnungspsalms (Ps 1,2: Memorierung und Meditierung der Tora JHwhs, dazu Weber, „Beitrag“; Weber, „Psalm 1“) und anderes mehr weisen in dieselbe Richtung.

11 Vgl. etwa Childs, „Titles“; Slomovic, „Understanding“, ferner mit generelleren Überlegungen Wilson, Editing; Bayer, „Titles“. Neueren Datums sind die Überlegungen in Rösel, Redaktion, S. 164-170; Janowski, „Hindin“, II.1.b.

12 Vgl. Kleer, Sänger, S. 9-127; Johnson, David.

13 Bei einer monographisch angelegten Gesamtuntersuchung der Seitenbezüge wären die poetisch geformten Psalmtexte stärker einzubeziehen, als dies im Rahmen dieses Essays möglich ist.

14 Zur Methodik und Durchführung (am Psalter) vgl. Ballhorn, Telos; Weber, „Herr“; Weber, „Psalm 78“, S. 305-308. 
sich die Vermutung, dass die Einstellung der biographischen David-Psalmen in den Psalter (oder das Zuwachsen dieser Präskript-Angaben) weder als beliebig anzusehen ist, noch - so die gängige Annahme - allein durch vom jeweiligen Psalm ausgehende Assoziationen gesteuert ist. Vielmehr sind zugleich von Buchkontext und Leserichtung eincodierte Bedeutungszuschüsse in Rechnung zu stellen. Ein derart im Psalter aufgespanntes „Netzwerk“ generiert theologisch-spirituelle Sinnhorizonte, die über den jeweiligen Einzelpsalm hinausreichen und in memorierend-schriftkundiger Weise von Rezipierenden abgeschöpft werden (können). Angesichts des noch weithin unbeschrittenen Terrains ist ein heuristischer Charakter der Untersuchung und damit Diskussionsbedarf angezeigt.

Die dreizehn Psalmen mit ihren biographischen Präskript-Angaben und den (wichtigsten) Referenzhorizonten ${ }^{15}$ werden zunächst tabellarisch aufgeführt. Beigegeben sind Ps 2 und 72, nicht zuletzt aufgrund ihrer buchstrukturellen wie theologischen Bedeutung. ${ }^{16}$

Wir lassen bei unseren Ausführungen Ps 142, den einzigen Psalm mit David-Biographie innerhalb der (späteren) Psalterteilbücher IV+V, ausser acht und konzentrieren uns auf die Belege innerhalb der ersten beiden David-Sammlungen. ${ }^{17}$ Ein erste Sichtung der verbleibenden zwölf situativen Präskript-Angaben ergibt, dass mit acht Belegen (Ps $51 ; 52 ; 54 ; 56 ; 57 ; 59 ; 60 ; 63$ ) der Schwerpunkt in der zweiten David-Sammlung (Ps 51-72*) liegt, während die erste Sammlung (Ps 3-41*) lediglich vier Angaben (Ps 3; 7; 18; 34) aufweist.

\footnotetext{
$15 \mathrm{Zu}$ Aufweis und Diskussion weiterer (möglicher) Verweistexte vgl. Kleer, Sänger, S. 26-28.87-110; Johnson, David, ferner Janowski, „Hindin“ (I.1.e + II.1./3. + Anhang).

16 Verwendete Abkürzungen: $1 \mathrm{~S}=1 \mathrm{Sam} ; 2 \mathrm{~S}=2 \mathrm{Sam} ; \mathrm{D}(+)=$ David (Zuschreibung) $(+=$ zusätzlich [vorangehend]: „zugehörig dem Knecht JHwHs"); Natan I = erstes Erscheinen Natans vor David (2 Sam 7); Natan II = zweites Erscheinen Natans vor David (2 Sam 12); nK = nach Königsantritt; $\mathrm{S}$ = Salomo (Zuschreibung); vK = vor Königsantritt.

17 Das biographische Präskript von Ps 142 speist sich aus Bestandteilen der Präskriptangaben zu Ps 63 und Ps 57 (in der LXX haben auch die nachfolgenden Psalmen 143 und 144 David-biographische Präskript-Angaben). Der Psalm hat seinen Ort in der letzten DavidGruppe (Ps 138-145), die ältere (David-)Psalmen rezipiert und adaptiert (dazu Buysch, Davidpsalter). Die Psalterteilbücher IV+V zeichnen sich durch ein verändertes David-Bild aus, das weniger biographisch-situativ (retrospektiv) als ideal bzw. endzeitlich (prospektiv) ist. Anzumerken bleibt, dass die aus Teilen von Ps 57 und Ps 60 zusammengefügte Komposition Ps 108 weder die eine noch die andere Angabe zur David-Biographie aufnimmt, sondern sich mit dem Hinweis „zugehörig David“ begnügt.
} 


\begin{tabular}{|c|c|c|c|c|}
\hline 2 & - & - & $\left(2 S_{7,14}\right)$ & nK (Natan I) \\
\hline 3 & $\mathrm{D}$ & $\begin{array}{l}\text { „bei seinem Fliehen vor } \\
\text { Absalom, seinem Sohn“ }\end{array}$ & ${ }_{2} \mathrm{~S}_{15^{-18}}$ & nK (Absalom) \\
\hline 7 & $\mathrm{D}$ & $\begin{array}{l}\text { „wegen der Worte Kuschs, } \\
\text { des Benjaminiters“ }\end{array}$ & $2 \mathrm{~S}_{18-19}(?)$ & nK (Absalom) \\
\hline 18 & $\mathrm{D}+$ & $\begin{array}{l}\text { „an dem Tag, als JHWH ihn } \\
\text { rettete aus der Hand aller } \\
\text { seiner Feinde und aus der } \\
\text { Hand Sauls“ }\end{array}$ & $\begin{array}{l}1 \mathrm{~S}_{17-2 \mathrm{~S}} 22 \\
{\left[2 \mathrm{~S}_{7,1}\right]}\end{array}$ & $\begin{array}{l}\mathrm{nK}(\text { alle })+ \\
\mathrm{vK}(\text { Saul })\end{array}$ \\
\hline 34 & $\mathrm{D}$ & $\begin{array}{l}\text { "bei seinem Verstellen } \\
\text { seines Verstands vor Abimelech, } \\
\text { er ihn vertrieb und er [weg]ging“" }\end{array}$ & $1 S_{21-22 / 23}$ & $\mathrm{vK}($ Saul) \\
\hline $5^{1}$ & $\mathrm{D}$ & $\begin{array}{l}\text { „als Natan, der Prophet, zu ihm } \\
\text { ging, nachdem er gegangen war } \\
\text { zu Batseba“ }\end{array}$ & $2 S_{11-12}$ & nK (Natan II) \\
\hline $5^{2}$ & $\mathrm{D}$ & $\begin{array}{l}\text { „beim Gehen Doëgs, des } \\
\text { Edomiters, er Saul berichtete } \\
\text { und zu ihm sprach: ,David ist } \\
\text { gegangen zum Haus } \\
\text { Ahimelechs“ “ }\end{array}$ & ${ }_{1} S_{21-22}$ & vK (Saul) \\
\hline 54 & $\mathrm{D}$ & $\begin{array}{l}\text { „beim Gehen der Sifiter und } \\
\text { sie zu Saul sprachen: } \\
\text { „Hält sich David etwa nicht } \\
\text { verborgen bei uns?!““ }\end{array}$ & $1 \mathrm{~S}_{23}(-26)$ & vK (Saul) \\
\hline $5^{6}$ & $\mathrm{D}$ & $\begin{array}{l}\text { „als ihn ergriffen die Philister } \\
\text { in Gat“ }\end{array}$ & $1 \mathrm{~S}_{21}(27)$ & vK (Saul) \\
\hline 57 & $\mathrm{D}$ & $\begin{array}{l}\text { "bei seinem Fliehen vor Saul } \\
\text { in die Höhle“ }\end{array}$ & ${ }_{1 S} 24(22)$ & vK (Saul) \\
\hline 59 & $\mathrm{D}$ & $\begin{array}{l}\text { „beim Senden Sauls und sie } \\
\text { das Haus bewachten, um ihn } \\
\text { zu töten“ }\end{array}$ & 1S 19 & vK (Saul) \\
\hline $6 o$ & $\mathrm{D}$ & $\begin{array}{l}\text { „beim Kriegführen mit Aram- } \\
\text { Naharajim und Aram-Zoba, und } \\
\text { als Joab zurückkehrte und Edom } \\
\text { schlug im Salztal, zwölftausend } \\
\text { [Mann]“ }\end{array}$ & $\begin{array}{l}2 \mathrm{~S} 8(10) \\
(/ / 1 \text { Chr } 18[-19])\end{array}$ & $\begin{array}{l}\mathrm{nK} \\
\text { (Nachbarstaaten) }\end{array}$ \\
\hline
\end{tabular}




\begin{tabular}{|c|c|c|c|c|}
\hline Psalm & Person & Situierung & Bezüge (1/2 Sam) & Bemerkungen \\
\hline 63 & $\mathrm{D}$ & $\begin{array}{l}\text { „bei seinem Sein in der Wüste } \\
\text { Juda“ }\end{array}$ & $\begin{array}{l}2 \mathrm{~S}_{15}^{-17} \\
\left(1 \mathrm{~S}_{23}^{-25}\right)\end{array}$ & $\begin{array}{l}\mathrm{nK} \text { (Absalom) } \\
\text { (vK Saul) }\end{array}$ \\
\hline 72 & $\begin{array}{l}S \\
{[D]}\end{array}$ & $\begin{array}{l}\text { - } \\
\text { Kolophon: „zu Ende gekommen } \\
\text { sind die Klagebitten Davids, } \\
\text { Sohn Isaïs“ }\end{array}$ & $\left(2 S_{7,16}\right)$ & nK (Natan I) \\
\hline 142 & $\mathrm{D}$ & „bei seinem Sein in der Höhle“ & ${ }_{1} \mathrm{~S}_{24}(22)$ & $\mathrm{vK}($ Saul $)$ \\
\hline
\end{tabular}

Die Konzentration auf die zweite Sammlung verstärkt sich noch, wenn man bedenkt, dass die erste David-Sammlung 37 Psalmen mit (expliziter) DavidZuschreibung, die zweite dagegen lediglich 18 Psalmen umfasst. Innerhalb der David-Sammlung Ps 51-72* enthält also nahezu jeder zweite Psalm „zugehörig David“ zusätzlich einen biographischen Verweis, und zwar verdichtet im Bereich von Ps $51-63{ }^{18}$

\section{Ps 3-7: ein „David-Absalom-Midrasch“ in Anfangsstellung}

Der Erstbeleg biographischer Präskript-Angaben zu David hat seinen Ort in der Buchouvertüre Ps $1-3 \cdot{ }^{19}$ Er erscheint in der ersten David-Zuweisung eines Psalms, ja der ersten Überschrift überhaupt - mit andern Worten: an einem ausgesprochen bedeutsamen Ort mit Blick auf die implementierte Lesestrategie des Psalters. ${ }^{20}$ Nach der poetischen Gestalt von Ps 1-2 treffen die Lesenden mit dem Präskript zu Ps $3^{21}$ erstmals auf einen Metatext, dessen Bedeutungsevaluierung eine erhöhte Verstehensleistung einfordert. Die hintereinander gestellten Ps 2 und Ps 3 haben (u.a. aufgrund der Identifikation

18 Damit konform verläuft der Befund, dass Ps 51-65 ohne Ausnahme David-Zuweisungen enthalten, während im Schlussteil Ps 66-72 diese abnehmen und gleichsam „auslaufen“ (Ps 66; 67; 71 ohne David-Erwähnung im Präskript, Ps 72: „zugehörig/für Salomo“).

19 Zur näheren Begründung und der damit verbundenen Lesestrategie hinsichtlich des Psalters vgl. Weber, „Buchouvertüre“.

20 Dazu ausführlich Weber, „HERR“, S. 240-243.

21 Zu diesem Psalm vgl. Botha/Weber, „Song I/II“ (zum Absalom-Bezug II S. 284-288). 
des königlichen Gesalbten mit David) einige Gemeinsamkeiten. Zugleich wird durch die iuxtapositio ein scharfer Kontrast aufgebaut: In Ps 2 ist die Zuwendung des Himmelskönigs zum Zionskönig prophetisch beglaubigt und unstrittig; sie legitimiert seine Stellung und lässt ihn siegreich über alle Völker triumphieren. In Ps 3 dagegen ist die Heilszuwendung an den König in Frage gestellt (V. 2); er erfährt sich von vielen bedroht und bittet um Rettung. Der schroffste Gegensatz aber wird durch die Präskript-Notiz „bei seinem Fliehen vor Absalom, seinem Sohn" und dem dadurch aufgerufenen Texthorizont von 2 Sam 14-18 induziert: In Ps 2 ist der "Sohn Gottes“ auf dem „heiligen Berg“ inthronisiert und bekommt die Verheissung, die „Enden der Erden“ zu besitzen (V. 6-9). Dagegen erlebt man David in Ps 3 auf der Flucht vor "seinem Sohn“, weg vom Tempelberg, die Lade als Ort der Gegenwart Gottes zurücklassend. ${ }^{22}$ Nach der "Hoheitsmessianologie“ von Ps 2 zeigt Ps 3 (durch die nachfolgenden Psalmen verstetigt) einen gefährdeten, bedrängten, leidenden König, aber auch einen betenden, vertrauenden, sich bei Gott bergenden David. Von Ps 3 und seinem Präskript eingewiesen, zeichnen auch die weiteren davidischbiographischen Angaben weithin diesen David als für die Nachbetenden exemplarischen Menschen, der zur Identifikation einlädt.

David-Zuschreibungen werden in der Folge (Ps 4ff.) fortgesetzt, biographische Vermerke (vorerst) nicht. Ps 3 und das ihm beigegebene überschriftliche Verweissystem nimmt die Kopfstellung eines Clusters von „Klagebitten“ ein: Ps 3-7 bilden eine konsistente Gruppe von Psalmen aus (Feind-)Bedrängnis (innerhalb der grösseren Einheit Ps 3-14). ${ }^{23}$ Aufgrund dieser rezeptionssteuernden Signale darf angenommen werden, dass die biographische Präskribierung über Ps 3 nicht nur den unmittelbaren poetischen Folgetext, sondern Ps 3-7 insgesamt (und allenfalls weitere Psalmen) in den Horizont der Absalom-Rebellion gegen David einweist.

Diese Annahme erhält insofern Sukkurs, als das Präskript zu Ps 7, dem Schlusspsalm dieses gruppettos, wahrscheinlich ebenfalls auf diesen Geschehenszusammenhang verweist. Angesichts der kryptischen Angabe „... wegen der Worte Kuschs, des Benjaminiters" ist zwar keine Sicherheit zu gewinnen. Die Referenz auf Saul hat von den drei Verstehensoptionen ${ }^{24}$ allerdings den geringsten Textanhalt, während die andern beiden - in unter-

22 Vgl. Weber, „HERR.“ S. 248-251.

23 Zum Profil von Ps 3-14 (und darin Ps 3-7) vgl. Hartenstein, „Recht“.

24 Zur Darlegung und Diskussion der Optionen „Saul“ (so schon Targum, Talmud, vgl. 1 Sam 24,8-22), „Schimi“ (vgl. 2 Sam 16,5-13; Est 2,5) und „ein Kuschiter“ (der David die Todesnachricht Absaloms überbrachte, vgl. 2 Sam 18,19-32; 1 Kön 2,44) vgl. Millard, Komposition, S. 131f.; Kleer, Sänger, S. 87-90; Johnson, David, S. 131-139. 
schiedlicher Weise - auf den David-Absalom-Konflikt abstellen (Schimi: 2 Sam 16,5-13, ein Kuschiter: 2 Sam 18,19-32). ${ }^{25}$ Kommt dazu, dass der vom Präskript zu Ps 3 her aufgespannte Verstehenshorizont eine Leseoption im Zusammenhang des David-Absalom-Konflikts präferiert. Damit ist wahrscheinlich gemacht, dass die „Eckpsalmen“26 der Kleingruppe Ps 3-7 auf David-AbsalomKonfliktkonstellationen (2 Sam 15-18/19) verweisen und die Psalmengruppe insgesamt in diesen Verstehenshorizont stellen. Entsprechend lässt sich von einem „David-Absalom-Midrasch“27 sprechen.

Weshalb es zu diesem Konflikt im Hause Davids kam, bleibt zu Beginn der ersten David-Sammlung unerörtert. Die Antwort wird später, zu Beginn der zweiten David-Sammlung - jedenfalls andeutend - nachgetragen: mit Ps 51, seinem Präskript und darin angesteuerten Natan-Aussagen aus den Samuelbüchern (s.u.). Auch die Frage, warum der Psalter zu Beginn nicht den zeitlich vorangehenden Saul-David-, sondern den späteren David-AbsalomKonflikt aufgreift, wird kaum allein mit Hinweis auf die in Ps 3 sich bietenden Begriffsassoziationen zu beantworten sein. Die Antwort dürfte mindestens so sehr in der angestrebten Polarität von Inthronisierung/Autorisierung (Ps 2) und Throngefährdung (Ps 3) liegen. Jedenfalls wird bereits am Psalterbeginn erkenntlich, dass die Erzählsequenz der David-Geschichte nicht das wesentliche Kriterium in der Anordnung der Präskript-Angaben zur vita David abgab.

25 Millard, Komposition, S. 131f., und Kleer, Sänger, S. 89, plädieren für den Verweis auf den Kuschiter. Millard versteht dabei כושי nicht als Eigenname, sondern als Volksbezeichnung und schreibt: „In der gesamten deuteronomistischen Davidsgeschichte wird nur an einer Stelle ein Kuschiter erwähnt: 2 Sam 18,21ff. Ein Kuschiter, also ein vermutlich versklavter Neger, den die Überschrift von Ps 7 dem Stamme Benjamin zuordnet, wird als Überbringer der schlechten Nachricht für David vom Tode seines Sohnes Absalom ausgewählt." Allerdings wäre dann im Präskript von Ps 7 (mit der griechischen Texttradition) כושי zu ändern. Die Bezeichnung eines fremdländischen Kuschiters zugleich als Benjaminiter wirft aber Fragen auf, die auch Barbiero, Psalmenbuch, S. 81-84, und Janowski, „Hindin“, II.1.b, nicht auszuräumen vermögen (der Vorschlag, בנ־ימיני nicht als Eigenname, sondern als „Einwohner des Südens“ [Arabien, Jemen] zu lesen, wirkt gesucht). Ich tendiere zu einem Schimi-Bezug (vgl. sub voce עפר Ps 7,6 mit 2 Sam 16,13, ferner 2 Sam 22,43 $\approx$ Ps 18,43).

26 Zur Verklammerung vgl. etwa sub voce קום Ps 3,2.8 mit Ps 7,7 einerseits sowie 2 Sam 15,14; 17,1 mit v.a. 18,31f. (Kuschiter) andererseits, ferner מגן Ps 3,4; 7,11; ישע/ישועה Ps 3,8f.; 7,2.

27 Zur legitimen Verwendungsweise des Begriffs Midrasch vgl. Childs, „Titles“, S. 149f.; Slomovic, „Understanding“, S. 352-355. 
Es ist denkbar, dass der mit Ps 3-7 aufgespannte Horizont des David-AbsalomKonflikts in der Fortlesung der weiteren Psalmen zunächst erhalten bleibt. Da in der Folge aber keine weitere biographische Notiz nachgereicht wird, dürfte er zunehmend verblassen. Mit Ps 15 beginnt die zweite, bis Ps 24 laufende Teilkomposition, in deren Mitte die Psalmen 18-21 stehen: Königspsalmen (Ps 18; 20f.), die den Tora-Psalm 19 rahmen. ${ }^{28}$ Darin setzt Ps 18 und sein Präskript einen neuen, stark davidisch-biographischen Akzent. Angezeigt im Präskript und durchgeführt im Psalm rückt gegenüber dem Not-bestimmten „David-Absalom-Midrasch“ Ps 3-7(ff.) eine deutlich andere Perspektive in den Vordergrund: die Bezeugung von JHWHs Retten, dem entsprechend Lobdank (תודה) gebührt (vgl. über das Präskript hinaus v.a. V. 4.17-20.41-51). Mit Ps 18 wird die Not als endgültig gewendet bezeugt. Denn mit Davids Worten, „an dem Tag, als JHWH ihn rettete aus der Hand aller seiner Feinde und aus der Hand Sauls“ wird ein finaler Aspekt eingebracht. Die Schlussstellung im Pendent 2 Sam 22 ( $\approx$ Ps 18 ) unterstreicht das abschliessend-summarische Moment und damit den testamentarischen Charakter, der diesem Königslobdank zukommt. ${ }^{29}$ Die Doppelüberlieferung, also die „Hereinnahme“ von 2 Sam 22 als Ps 18 in toto, ${ }^{30}$ zeigt die Schlüsselbedeutung dieses Psalms. Über die David-Zuweisungen und die biographischen Angaben hinaus markiert Ps 18 eine ausgesprochen starke Rückbindung an die Samuelbücher. ${ }^{31}$ Mit dem gegenüber 2 Sam 22 justierten und unter Einbezug von לעבד יהוה und damit in Anlehnung an Mose (vgl. Dtn 34,5; Jos 1,1.13.15 u.a.) verstärkt autorisierten Präskript zu Ps 18 wird eine neue,

28 Vgl. Weber, Werkbuch III, S. 161-163.

29 Vgl. dazu auch die Endzeile in V. $5^{1}$ und den Reflex auf die Natan-Verheissung (2 Sam 7,12f.). Die Bezeichnung השירה הזאת hat Anklänge zu Moses testamentarischem Lied (vgl. Dtn 31,19.21f.30; 32,44). Zur Doppelung von 2 Sam 22 und Ps 18 vgl. v.a. Kleer, Sänger, S. 11-35 (mit Priorisierung der gestaffelt entstandenen Fassung im Psalter). Er verweist zudem auf eine Verbindung zwischen den Präskripten von Ps 18 und Ps 7 - also den beiden Psalmen, die mit Blick auf David-biographische Überschriften aufeinander folgen: Nur in diesen beiden Fällen wird die biographische Notiz mit Relativpartikel an die David-Zuschreibung angeschlossen und David damit explizit als Sprechender ausgewiesen (S. 11).

30 In der (kanonisch gewordenen) Leseabfolge sind die Samuelbücher (aus den Nebiim) dem Psalter (aus den Ketubim) vorgeordnet, unabhängig davon, ob 2 Sam 22 oder Ps 18 die ältere Fassung bietet.

31 Dies umso mehr, als 2 Sam 22 bereits bei Ps 3 (ff.) als Hintergrundfolie präsent ist (zu diesem Sachverhalt, der hier nicht ausgeführt werden kann, vgl. Weber, „HERR“, S. 240-248; Botha/Weber, „Song II“, S. 284.288-290; Weber, „Danklied“). 
prägnante Lesespur eröffnet: Die David-Psalmen sind nicht allein auf dem Hintergrund artikulierter Nöte und Feindbedrängnisse zu lesen, ${ }^{32}$ sondern auch schon im Licht der endgültigen Rettung JHWHs (Lobdank-Einfärbung). Derart wird die Natan-Verheissung unter Rückgriff auf die Samuelbücher "bestätigt" (2 Sam 7,16, dazu Ps 18,51). Zugleich werden (in Umgreifung des „David-Absalom-Midrasch“) Aussagen des vorangestellten Ps 2 reaktualisiert (V. 6-9).

Die von Gott geschenkte Rettung Gottes aus der Hand von Davids Widersachern wird im Präskript 2 Sam 22,1 $\approx$ Ps 18,1 als zweifache dargestellt. Dabei fällt auf, dass zunächst "alle seine Feinde“ und anschliessend erst „Saul“ angeführt wird. Diese Anordnung ${ }^{33}$ lässt erkennen, dass die zeitliche Abfolge nicht im Vordergrund steht. ${ }^{34}$ Aufgrund der herausgehobenen Bedeutung von Ps 18 im Buch lässt sich erwägen, ob mit der zweigeteilten Nennung der Widersacher ein katalysierender bzw. regulativer Impuls verbunden ist. Ps 18 fungiert dann gleichsam als „Drehscheibe“: Nachdem von Ps 3-7 (Absalom) in der Diktion der Überschrift von Ps 18 "alle seine Feinde“ zur Sprache kamen (Analepse), rücken nachfolgend die Bedrängnisse durch Saul in den Vordergrund (Prolepse). So gesehen wird der Horizont des nachfolgenden Ps 34 (sowie Ps 52-59) vorbereitet.

Von Ps 18 bis zu Ps 34, dem nächsten mit David-Biographie, wird in der Leseabfolge ein beträchtlicher Weg zurückgelegt. An die Teilkomposition Ps 15-24 schliesst sich als dritte Ps 25-34 an (Rahmung mit alphabetischen Akrosticha). Ps 34 nimmt darin die Endposition ein. Es fällt auf, dass nach der seit Ps 3 (nahezu $\left.{ }^{35}\right)$ ungebrochenen David-Zuweisung im Vorgängerpsalm 33 eine solche unterbleibt. Wie immer man diese Präskript-Absenz zu erklären hat, sie führt im Leseprozess zu einer verstärkten Wahrnehmung der DavidZuscheibung über Ps 34. Erstmals (nach dem Hinweis in Ps 18,1) wird ein Psalm mit einer Episode aus dem Saul-David-Konflikt verbunden (ähnlich nachher Ps 52 und v.a. Ps 56). Die Wendung שנה את־טעם ist in 1 Sam 21,13 und Ps 34,1 identisch. In der David-Erzählung ist das Gegenüber allerdings der philistäische Stadtkönig Achisch und nicht Abimelech (oder allenfalls Ahimelech).

32 Alle übrigen Präskripte - mit Ausnahme von Ps 60 - und überwiegend auch die damit verbunden Psalmen sprechen eine (aktuelle, nicht [restlos] behobene) Notlage an und artikulieren Gebetsworte aus Feindbedrängnis.

Kommt Saul durch die separate (nachgestellte) Erwähnung eine Sonderstellung zu (aufgrund seiner Königswürde als Gesalbter JHWHs?)?

34 Unbesehen davon, wer unter "alle seine Feinde“ einzuschliessen ist, ist Saul zeitlich nicht der letzte, sondern - von Goliat abgesehen - der erste Widersacher.

35 Das Fehlen von Präskript-Vermerken über Ps 10 lässt sich aufgrund der Zusammengehörigkeit von Ps 9-10 (vgl. LXX) als alphabetisches Akrostichon erklären. 
Für diesen Umstand gibt es verschiedene Erklärungsversuche, die uns hier aber nicht weiter zu beschäftigen brauchen. ${ }^{36}$ Möglicherweise ist die Irritation, welche die „unpassende“ Präskriptangabe auslöst, ein Hinweis auf multiple Texthorizonte. ${ }^{37}$ Dies fügt sich zum weisheitlich-belehrenden Stil von Ps 34 (im Kern ein Lobdank). ${ }^{38}$ Er greift ein Moment aus der vita David heraus, das im Überschriftssystem einzigartig ist, arbeitet aber gleichwohl dem späteren „David-Saul-Cluster“ (Ps 52-59) zu.

Mit Blick auf die erste David-Sammlung (Ps $\left.3-41^{*}\right)$ haben wir aufgrund der biographischen Angaben demnach zwei Konflikt-Konstellationen, die freilich distanziert platziert sind: gleich am Anfang die Bedrängnis durch seinen Sohn Absalom (Ps 3-7) und am Ende der dritten Teilsammlung ein Verweis auf den Konflikt mit Saul (Ps 34). ${ }^{39}$ „Dazwischen“ figuriert Ps 18 ( 2 Sam 22), nach vorn wie nach hinten ausstrahlend und entsprechend die biographischen Lesehinweise in der ersten David-Sammlung mitprägend.

Im Bereich Ps 51-63 sind Psalmen mit biographischen Präskript-Komponenten in der Mehrheit. ${ }^{40}$ Ps $5^{1}$ (verknüpft mit dem vereinzelten asaphitischen Ps 50), der die zweite David-Sammlung eröffnet, steht eigenständig voran und ist gesondert zu erwägen (dazu s.u.). Die nachfolgenden Ps $55^{2-55}$ sind formkritisch

${ }_{3} 6$ Zur Diskussion vgl. Johnson, David, S. 69-76. Über den von ihr erwogenen Assoziationshorizont zu Abimelech, dem König von Gerar (vgl. v.a. Gen 20) hinaus ist auch Abimelech, Gideons Sohn und Richter/König in Israel (vgl. Ri 9, ferner 2 Sam 11,21 [Davidzeit!]) in Erwägung zu ziehen (vgl. zum Motiv der Vertreibung sub voce גרש Ri 9,41; Ps 34,1). Auch eine Verschreibung von Ahimelech zu Abimelech ist denkbar (so 1 Chr 18,16), zumal die Begegnung Davids mit Achisch (2 Sam 2,11-16) gerahmt wird durch diejenige mit Ahimelech, dem Priester in Nob, sowie dem sich daraus ergebenden Ergehen des Priesters (vgl. 2 Sam 21,2-10; 22,6-23, dazu die Erwähnung im Präskript von Ps 52). In diesem Fall wäre eine Assoziierung mit 1 Sam 21f. insgesamt im Fokus der Überschrift zu Ps 34 .

37 Die Schlussangabe וילך, ,und er ging [weg]“ (Ps 34,1), die den engeren Kontext auf nachfolgende Geschehnisse hin überschreitet (wie dieselbe Wendung in 1 Sam 22,1.3.5; 23,5 zeigt), weist in ähnliche Richtung.

38 Ein solcher ist in abgeschwächter Form auch für Ps 18 anzunehmen (neben dem quasitestamentarischen Charakter vgl. namentlich V. 26-28).

39 Die letzte Teilsammlung Ps 35-41 bleibt ohne biographische Hinweise (vgl. aber die Angabe להזכיר zur Erinnerungsstiftung“" o.ä. im Präskript zu Ps 38).

40 Zur Substruktur der zweiten David-Sammlung vgl. Weber, Werkbuch I, S. 233ff.; Hossfeld/ Zenger, Psalmen 51-100, S. 27f. 
(„Klagebitten“) und thematisch als Kleingruppe von „Feindpsalmen“ erkennbar. Alle vier Psalmen werden in der Überschrift als משכיל charakterisiert, wohl in der Bedeutung „Lehrstück“ o.ä. ${ }^{41}$ Daran schliesst sich die Kleingruppe Ps 56-6o an. Gegenüber Ps 52-55 ist sie formal etwas disparater, wird aber durch den Vermerk מכתם verklammert.42 Die Angabe dürfte einen Texttyp bezeichnen, ihre Bedeutung ist unsicher („Auf-, Inschrift" = מכתב?). ${ }^{43}$ Der Kern Ps 57-59 (ohne die rahmenden Ps 56 und Ps 6o) hat zudem die PräskriptAngabe אל־תשחת,Vertilge nicht!

Gleicht man diese Beobachtungen mit solchen zur David-Biographie ab, bestätigt sich die Kompaktheit der Doppelgruppe Ps 52-55|56-6o, die durchgängig von „Feind-Bedrängnis“ geprägt ist. ${ }^{45}$ In den Präskripten zu diesen neun Psalmen findet sich nicht nur die grösste Dichte an Hinweisen zur vita David (+-+-|++-++), sondern diese führen auch allesamt - mit Ausnahme von Ps 60 in Endstellung - in dieselbe Konflikt-Konstellation zwischen Saul und David (also in die Zeit vor Davids Königsantritt). Von daher lässt sich hinsichtlich Ps 52-59 von einem "Saul-David-Midrasch“ sprechen. ${ }^{46}$ Angesteuert werden unterschiedliche Episoden im Bereich von 1 Sam 19-27, mit Schwergewicht in 1 Sam 21-24 (s.o., Tabelle). Die Abfolge der Hinweise folgt freilich (auch hier) nicht genau der Erzählspur von 1 Sam. Die Anordnung kann sich von den

41 Sonst in Psalter-Präskripten noch über Ps $32 ; 42 ; 44 ; 45 ; 47 ; 74 ; 78 ; 88 ; 89 ; 142$ (ebenfalls mit Angabe zur David-Biographie). Abgesehen von Ps 32 und 142 werden die übrigen Stücke nicht David zugewiesen, sondern mit Sängergilden in Verbindung gebracht.

42 Sonst in Psalter-Präskripten nur noch über Ps 16.

43 Allenfalls eine Weihinschrift (Epigramm). Der Verweis im Präskript von Ps 57 auf Davids Flucht „in die Höhle“ lässt als mögliche Analogie an die Höhlen-Inschriften von Chirbet Bet Lay (in der Nähe von Lachisch) denken (dazu Renz, Inschriften 1, S. 242-251).

Im Psalter sonst nur noch im Präskript des asaphitischen Ps 75. Mit Kleer, Sänger, S. 102, lässt sich erwägen, ob damit ein (zusätzlicher) Reflex auf die Saul-David-Auseinandersetzung eingetragen ist: die Anweisung Davids, Saul zu verschonen (1 Sam 26,9).

45 Die Bearbeitung der Notlagen vollzieht sich in Formen direkter Feindanklage, vor allem aber betend mittels Klage- und Bittgebeten, schliesslich auch reflektierend-weisheitlich und verkündigend vor der Gemeinde. In der zweiten Kleingruppe tritt zur Klagebitte (תפלה) gelegentlich der Lobdank (תודה). Zum Profil der beiden Kleingruppen vgl. auch Süssenbach, Psalter, S. 99-127.

46 Angesichts des aufgespannten Saul-David-Bezugs werden bei der Rezeption auch die (eingeschobenen) David-Psalmen ohne biographische Angaben (Ps 53 [ Ps 14]; 55; 58) assoziativ in die Nähe dieses situativen Geschehens gerückt. Für Ps 53 (Hossfeld/Zenger, Psalm 51-10o, S. 79) und Ps 58 (Krawczack, Gott, S. 408-418) wird eine spätere Einstellung in die genannten Kleingruppen erwogen. 
einzelnen Psalminhalten her aufgedrängt haben, zudem bietet die Konfiguration im Buch gewisse Indizien. ${ }^{47}$

Die biographischen Präskript-Komponenten steuern den Leseprozess dahingehend, die in Ps 52-59 poetisch artikulierten Konflikte im Licht von (spezifischen) Saul-David-Auseinandersetzungen aus dem Erzählwerk von 1 Sam zu sehen. Wie schon im durch die Präskripte Ps 3 und/bis 7 generierten ersten Midrasch bilden Bedrängnis-Konstellationen auch bei diesem zweiten das verbindende Band. Anders als bei Ps 3-7, wo die Not im Gebet vor Gott (תפלה) bewältigt wird, ${ }^{48}$ lassen die "Gattungsangaben“49 im Verbund mit Hinweisen in den Psalmkorpora ${ }^{50}$ für Ps 52-59/6o eine Transformierung im Sinne einer pädagogisch-didaktischen (Neu-)Verwendung annehmen. Eine belehrende Funktion haben im Vorfeld bereits der weisheitliche Ps 34 - in seinem Präskript ebenfalls auf den Saul-Konflikt verweisend - sowie in anderer Weise Ps 18 mit seiner Überschrift. Dessen Angabe lässt die (biographisch markierten) Konfliktpsalmen „vom Ende her" und d.h. bereits im Horizont der (verheissenen) JHWH-Rettung verstehen. Im Zuge seiner Einbettung in den Psalter als Ps 18 bringt dieser damit einen zweiten Interpretationshorizont hinzu.

47 In den ersten beiden Präskripten (Ps $52 ; 54$ ) wird auf „(verhängnisvolles) Wortgeschehen“ (eingespielte Zitate, vgl. namentlich Ps 54,2 mit 1 Sam 23,19), in den übrigen drei (Ps 56; 57; 59) auf Sachverhalte verwiesen. Hinsichtlich Ps 52 und 54 beachte man auch die Ähnlichkeiten in Formulierung, Struktur und Inhalt zwischen den je mit בבוא beginnenden Präskript-Angaben zur David-vita. Alle mit Ausnahme von Ps 56 in der „Mitte“ erwähnen Saul übrigens explizit. Ps 52, der den „Saul-David-Midrasch“ eröffnet, weist gewisse Bezüge zum Präskript von Ps 34 auf, das die erste biographische Saul-Referenz bietet (vgl.

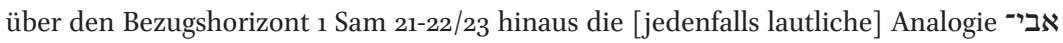
(מלך / אחימלך Ähnliches gilt vom Präskript zu Ps 56 hinsichtlich Ps 34 (und Ps 52). Die Angaben zu Ps 59, dem letzten Psalm innerhalb des „Saul-David-Midraschs“, greifen zeitlich am weitesten zurück (1 Sam 19 - ob sich mit Blick auf die beiden Frauen Michal und Batseba eine Klammer zwischen Ps 51 und 59 ergibt?). Vgl. dazu die Hinweise von Johnson, David, zu den jeweiligen Psalmen und ihren Bezügen, ferner Janowski, „Hindin“, II.3.

48 Aufgrund der Überschriftsangaben in Ps 3-7 und einigen Hinweisen in den (Eck-)Psalmen selbst (vgl. Ps 3,9; 7,18) ist (sekundär) allenfalls ein liturgischer Rahmen anzunehmen.

49 Für משכיל ist dies sicherer als für מכם, wobei auch für die zweite Angabe eine die Memorierung anstossende und damit didaktische Funktion zu erwägen ist. Zu ללמשיל מש מi in Ps 60,1 und der Einordnung von Ps 60 und seinem Präskript s.u.

5o Vgl. dazu Ps 53 ( $\approx$ Ps 14); 58,4-6.11f. 
Im Fortlauf des Psalters (lectio continua) werden im Teilbuch I die DavidPsalmen nur kurzzeitig unterbrochen (Ps 10; 33), in Teilbuch II dagegen setzen sie angesichts eines längeren Blocks an Sängergilden-Psalmen (Ps 42-49; 50) eine Zeit lang ganz aus. Mit Ps 51, dem ersten David-Psalm der zweiten DavidSerie, ist dadurch ein betonter Neueinsatz gegeben (dazu s.u.). Er ummantelt zusammen mit Ps 60 den „Saul-David-Midrasch“ Ps 52-59. Ps 60 erweist sich substrukturell und aufgrund der Typisierung als מכתם zur Gruppe Ps 55-59 zugehörig und bildet einen modifizierten Abschluss derselben. Dabei geht die individuelle Klagebitte von Ps 59 („Ich-Rede“) zu einer kollektiven in Ps 60 („Wir-Rede“) über. Dem entspricht, dass der Saul-David-Zusammenhang verlassen wird und im Präskript nationale Konflikte aus der Königszeit als Interpretationshorizonte angezeigt sind. ${ }^{51}$ Aufgrund der Erwähnung militärischer Konfrontationen mit drei Nachbarmächten erweist sich diese PräskriptAngabe als anschlussfähig zur Angabe „aus der Hand aller seiner Feinde“ im Präskript von Ps 18. Nimmt man das anschliessende „und aus der Hand Sauls“ hinzu, lässt sich die Gruppierung Ps 52-59/60 als invertierten Anklang an das zweiteilige Präskript von Ps 18 lesen. Bei der Fortlesung von Ps 52-59 (Saul) zu Ps 60 (Nachbarmächte) bleiben die dynastischen Konflikte im eigenen Haus ausgespart (dazu Ps 63 und v.a. Ps 51). Im Präskript zu Ps 60 fällt auf, dass zwischen David-Zuweisung (die anders als Ps 56-59 der מכתם-Charakterisierung nachfolgt) und biographischem Verweis singulär die Fügung ללמד zum zu lehren" eingeschoben ist. Mit dieser Zweckangabe wird die didaktische Einfärbung von Ps 52-59 am Gruppenende verstärkt und der VolksklageCharakter des Psalms (sekundär) in eine Belehrung transformiert. ${ }^{52}$ Im Licht dieser Präskript-Komponenten wird die eingelagerte Gottesrede (V. 8-11) hervorgehoben bzw. als erfüllt angesehen. Damit tritt ein vertrauensstärkendes Moment in den Vordergrund - ganz im Sinn von Ps 18 (inklusive Präskript) mit seiner Bezeugung der Rettung Davids von all seinen Feinden. ${ }^{53}$

Ps 63, der letzte Psalm mit biographischen Präskript-Hinweisen innerhalb der Psalterteilbücher I und II, hat den offensten Verweis: „bei seinem Sein in

51 Der primäre Verweis geht auf 2 Sam 8, wobei 2 Sam 10 (ferner 1 Chr 18-19) mit im Blick sein dürfte, vgl. dazu Kleer, Sänger, S. 102-106; Johnson, David, S. 122-131. Hinsichtlich Ps 52-6o liegt ein „Edomiter-Rahmen“ vor (vgl. Doëg, der Edomiter, in Ps 52,2 und die Erwähnung Edoms in Ps 6o,2).

52 Dazu Kleer, Sänger, S. 105 f.

53 Im Rahmen der Fortlesung vgl. diesbezüglich auch den Nachbarpsalm 61 (V. 7 f., vgl. $2 \operatorname{Sam} 7,16)$. 
der Wüste Juda“. Als Deutehorizonte von Davids Aufenthalten „in der Wüste“54 bieten sich der (länger dauernde) Konflikt mit Saul (vgl. v.a. 1 Sam 22,5; 23,1424,23) und die Flucht vor Absalom (vgl. 2 Sam 15,23-17,29) an. ${ }^{55}$ Letztere sind prägnanter und werden aufgrund der Rede vom „König“ in Ps 63,12, die an einen installierten Regenten denken lässt, in den Vordergrund geschoben. Der letzte biographische Psalm (abgesehen von Ps 142) rückt den Absalom-Bezug also nochmals in den Vordergrund und bildet dergestalt mit dem "David-AbsalomMidrasch" Ps 3-7 einen rahmenden Bogen. ${ }^{56}$

Mit dem Präskript zu Ps 51 als Erstpsalm der zweiten David-Sammlung liegt ein aus den „Feind“-Konstellationen herausfallendes und biographisch singuläres Moment vor. Zu dessen Verständnis ist vorab ein Blick auf den Schlusspsalm $72 \mathrm{zu}$ werfen. In dessen Überschrift steht (allein) die Angabe „zugehörig Salomo“. Ihm beigegeben ist folgendes Kolophon: „Zu Ende gekommen sind die Klagebitten Davids, Sohn Isaïs“. Wird der vorausgehende Psalm von seinem Kolophon her gelesen, legt sich nahe, ihn als "davidisch" aufzufassen und die Zugehörigkeitsanzeige im Sinne von „für Salomo“ einzugrenzen. ${ }^{57}$ Diese Deutung bewährt sich vom Inhalt des Psalms her (eine Art Vermächtnis des scheidenden Königs an seinen Sohn). Über die Verklammerung der Königpsalmen 2 (Betonung auf Königseinsetzung) und 72 (Betonung auf gerechter Herrschaft) ${ }^{58}$ hinaus ist ein Bezug von Ps 72 zum königlichen Lobdank - sei es in der Gestalt von Ps 18 oder 2 Sam $22^{59}$ - denkbar:

54 Die Wendung מדבר יהודה findet sich ansonsten nur noch in Ri 1,16. Davids Aufenthalte in Ödland und Wüsten verweisen gleichwohl auf judäische Gebiete (vgl. Ri 15).

55 Ps 63 selbst bietet sowohl Knotenpunkte der Vernetzung mit der Verfolgung durch Saul (vgl. Ps 63,8f. mit 1 Sam 23,14-17) als auch zur Flucht vor Absalom (zum „Durst“-Motiv vgl. Ps 63,2 mit 2 Sam 16,14; 17,29).

56 Mit Blick auf den Absalom-Horizont sind auch Ähnlichkeiten mit Ps 3 zu notieren (vgl. Ps 3,6 mit Ps 63,7.9, Ps 3,8 mit Ps 63,12, Ps 3,3.9 mit Ps 63,8).

57 Das Kolophon markiert den Abschluss der (zweiten) David-Sammlung (Ps 51-72*). Diese besteht zwar nicht durchgängig, aber doch mehrheitlich aus David-Psalmen einerseits und Kפלות „Klagebitten“ andererseits, so dass „Klagebitten Davids“ als Sammelbezeichnung gerechtfertigt erscheint. Der Einschluss auch der ersten David-Sammlung (Ps 3-42*, [noch] ohne die dazwischen figurierenden Gildenpsalmen: Ps 42-49 „Söhne Qorachs“, Ps 50 „Asaph“) ist wenig wahrscheinlich. Es wäre dann ein Zusammenzug (lediglich) der beiden David-Sammlungen im Blick, was aber zur "elohistischen Redaktion“ (Ps 42-83) quer läuft. Zur Diskussion vgl. Rösel, Redaktion, S. 52-55.

58 Vgl. Ps 72,11 mit Ps 2,8, auch אפסי־ארץ Ps 2,8; 72,17, ferner die mit (י) אשר formulierten Beglückwünschungen (Ps 2,12; 72,17).

59 Der Vermächtnischarakter von 2 Sam 22 lässt sich als Rekapitulation vor der Thronübergabe an Salomo, seinen Sohn, verstehen. 2 Sam 22,51 $\approx$ Ps 18,51 enden mit der Ausdehnung der Verheissung: „David und seinem Samen, für allezeit“ (vgl. 2 Sam 7,12f.). 
Sind Davids Feinde zu Ende gebracht bzw. ausgetilgt worden (sub voce כלה, vgl. Ps 18,38[f.], dazu 2 Sam 22,38f.), kommen auch seine (aus der Not adressierten) Klagebitten an ein Ende (כלו, Ps 72,20). ${ }^{60}$ Der Stand des Präskripts von 2 Sam 22 $\approx$ Ps 18 wird mit dem Kolophon gleichsam eingeholt und als erfüllt angesehen (vgl. auch 2 Sam 22,49-51 Ps 18,49-51).

Ps 51, in betonter Erststellung, bringt zuvor freilich eine neue Note hinzu: die Sünde Davids und seine im Psalm artikulierte Busse. Der Verweis auf Natan, den Propheten (V. 2), lässt dessen Worte vergegenwärtigen, die im Psalterkontext ihre eigene Kontur bekommen. Angesichts von Davids Sünde rekapituliert der Prophet als Gotteswort in 2 Sam 12,7, dass JHWH David zum König salbte (vgl. Ps 2,2.6f.) und ihn aus der Hand Sauls rettete (vgl. Ps 18,1!61). Als Strafe widerfährt ihm gemäss 2 Sam 12,11 Unheil im eigenen Haus (vgl. den „David-Absalom-Midrasch“ Ps 3-7, dazu den Fluch Simis in 2 Sam 16,8). Ps 18 mit seinem Präskript wird so einerseits bestätigt, andererseits wird am Anfang der zweiten David-Sammlung der Absalom-Konflikt zu Beginn der ersten David-Sammlung rückwirkend erklärt und innerhalb des Psalters zur Geltung gebracht. Aufgrund von Davids Busse in Ps 51 (vgl. 2 Sam 12,13) nimmt das Unheil in Davids Familie nicht überhand: Das Kind stirbt, aber von Batseba wird ihm ein (weiterer) Sohn geschenkt. Gereicht Davids erstes „Gehen zu Batseba" (Ps 51,2)62 zu Sünde und Tod, so sein zweites zum Trost: Salomo, der spätere Thronfolger, wird geboren (vgl. 2 Sam 11,1-12,25, ferner 1 Kön 1). Die davidischen Eckpsalmen $5^{1}$ (Batseba) und 72 (Salomo) werden korreliert. Möglicherweise wird zugleich ein Netz aufgespannt zwischen unterschiedlichen „Sohn“- bzw. Dynastie-Erwähnungen (vgl. Ps 2,7; 3,1; 72,1.20). Ps 2 und Ps 72, den Rahmen um beide David-Sammlungen bildend, nehmen auf die Königtumsverheissung Natans bei seinem ersten Erscheinen vor David Bezug (2 Sam 7) ${ }^{63}$ Ps 51 als Eröffnungspsalm der zweiten Sammlung verweist dagegen auf das zweite Kommen Natans zu David (2 Sam 12). ${ }^{64}$ Das singuläre biographische Präskript zu Ps 51 lässt sich derart am Plausibelsten mit dem besonderen Ort des Psalms innerhalb der David-Sammlungen erklären. Ps 18 einerseits

6o Vgl. ferner die gemeinsamen Motive Gerechtigkeit (vgl. Ps 18,21-25 mit Ps 72,1-3.7), Zuwendung zu den Armen (vgl. Ps 18,28 mit Ps 72,4.12-14) und Sieg über die Feinde (vgl. Ps 18,4-17-20.36-46 mit Ps 72,8-11).

61 Man beachte dieselbe Wendung (הציל מיד שאול) in 2 Sam 12,7 und Ps 18,1 (vgl. auch die Verwendung von יד anstelle des synonymen כריל מיד שר 2 Sam 22,1).

62 Zur Formulierung im Gegenüber zu 2 Sam 11,4 vgl. Kleer, Sänger, S. 95f.

63 Zum Rückbezug auf 2 Sam 7,12-16 vgl. v.a. Ps 2,7, ferner Ps 2,6.8(f.) sowie Ps 72,(11.)17 (dazu Barbiero, „Risks“, S. 88-90). Vgl. auch die Bezüge zwischen Ps 2,8 und Ps 72,8.

64 Gottes Verhalten im Falle einer Versündigung Davids wird freilich bereits bei der Dynastieverheissung angesprochen (2 Sam 7,14f.). 
sowie Ps $5^{1}$ und Ps 72 andererseits bringen in das Gefüge der Psalmen mit biographischen Angaben die wesentlichen hermeneutischen Verstehensebenen ein. Dabei spielen Bezüge auf die beiden Natan-Prophetien eine bedeutende Rolle.

\section{Auswertung und Schlussüberlegungen}

Die Untersuchung ergab mit Ps 3-7 und Ps 52-59 zwei biographische, assoziativ mit der narrativen Überlieferung in den Samuelbüchern verbundene PsalmenCluster, die wir als „David-Absalom-Midrasch“ und „Saul-David-Midrasch“ bezeichneten. Im ersten Fall geschieht dies mittels Flankierung zweier biographisch-präskribierter Psalmen (Verklammerung), im zweiten durch Häufung und Variierung an Episodenbezügen in den Überschriften (Staffelung). Unterstützt werden die Zuweisungshorizonte im Fall von Ps 3-7 durch eine begrifflich, thematisch und gattungstypisch enge Verzahnung der fünf Psalmen. Bei Ps 52-59/6o (Doppelgruppe), wo die Textsorten etwas weniger konsistent sind, wird die Kohäsion durch Komponenten des Präskripts (Gattungstypik) gefördert und zugleich ein didaktisches Moment beigefügt. Dies gilt noch verstärkt für Ps 6o, dessen biographisches Präskript die Perspektive auf nationale Konflikte hin wendet und derart die Gruppe beschliesst. Alle diese Psalmen, inklusive Ps 63, dessen Präskript nochmals auf den David-Absalom-Konflikt hindeutet, zeichnen sich dadurch aus, dass ihnen Feindbedrängnisse zugrunde liegen und die Gattung der Klagebitte (תפלה) überwiegt. Der vereinzelt platzierte Biographie-Psalm 34 nimmt das lobdankende und belehrende Moment von Ps 18 auf, verstärkt letzteres und arbeitet mit diesem und dem Saul-Bezug dem prägnanten Cluster Ps 52-59/60 vor. Die Psalmen 18; 51 und 72 (dazu Ps 2) nehmen hermeneutisch und buchstrukturell (Doppelung: Ps $18 \approx 2$ Sam 22, Anfangs- bzw. Endpositionierung: Ps 2; 51; 72) innerhalb dieses Gefüges eine Sonderfunktion ein. Sie lehren einerseits, die Psalmgebete lobdankend (תודה) vom geschichtlichen Ende her als Rettung JHwHs an David (und seinem Haus/Volk) zu verstehen (Ps $18 \approx 2$ Sam 22). Eingeklammert in Dynastieverheissung und -weitergabe (Ps 2; 72) bringen sie andererseits auch die durch Sünde ausgelöste Krise im Davidshaus zur Sprache (Ps 51), die ihren Ausdruck im Absalom-Konflikt zu Beginn (Ps 3-7) und am Ende (Ps 63) der biographischpräskribierten Psalmen findet. Eine signifikante Rolle kommt dabei den von Natan überbrachten Gottesworten bei dessen erstem (2 Sam 7, vgl. Ps 2; 18; 72 ) und zweitem Erscheinen vor David (2 Sam 12, vgl. Ps 51) zu. Im folgenden Schaubild sind wesentliche Ergebnisse überblicksartig dargestellt: 


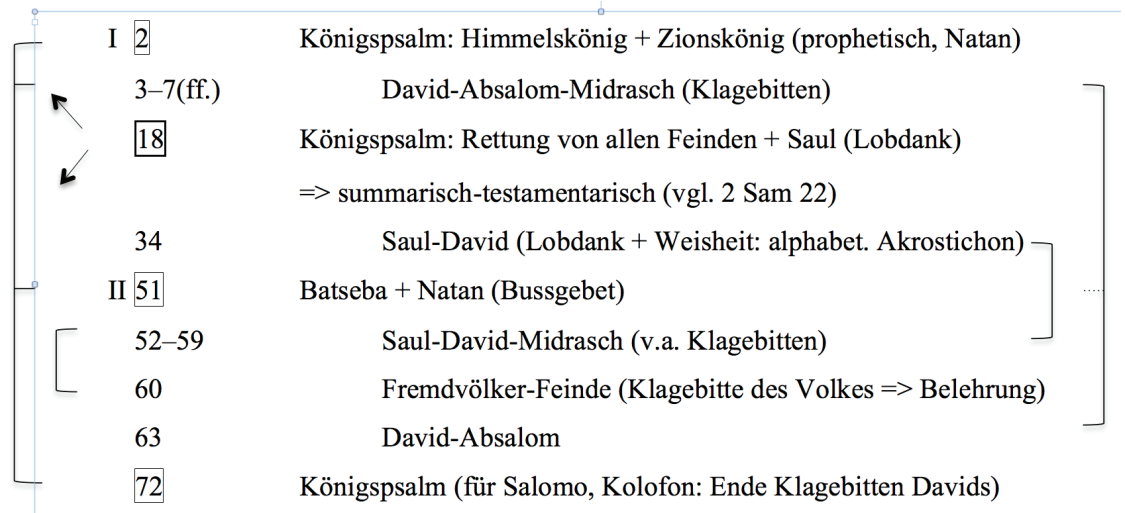

Obwohl die Platzierung der Psalmen mit davidisch-biographischen PräskriptKomponenten nicht dem Erzählverlauf der Samuelbücher entlang geht, ist ein buchstrukturelles Anordnungsmuster erkennbar. Damit ist die eingangs geäusserte Vermutung begründet, dass die Psalmen mit Angaben zur vita David weder beliebig eingestellt noch die von den Psalmkorpora ausgehenden Assoziationen das einzige Verstehenskriterium sind. Das vorliegende Ensemble biographischer David-Psalmen in den ersten beiden Psalterteilbüchern trägt in Verbindung mit der Assoziierung von Episoden aus den Samuelbüchern zur Generierung von Sinnzuschüssen bei. Die biographischen Angaben gehören im Verbund mit der David-Zuschreibung von Psalmen sowie der Doppelgestalt 2 Sam $22 \approx$ Ps 18 zu einem starken Verweissystem, das Samuelund Psalmenbuch aufeinander bezieht. Die damit einhergehende analeptische Struktur trägt ein spezifisches "Davidbild“ in den Psalter ein. ${ }^{65}$

Eine synchrone Fragestellung im Sinne einer akkumulierenden Sinnkonstituierung im Rahmen von Lese-, Memorierungs- und Meditierungsprozessen stand im Vordergrund dieser Studie. Die Erfassung der angezeigten Sinnpotenzen und Verstehenshorizonte bedarf einer Mehrfachlesung und setzt „Schriftkundigkeit“ voraus - ganz im Sinne des ins Buch einweisenden Psalms 1 (vgl.v.a.Ps1,2). Es ist davon auszugehen, dass unter Einbezug weiterer Daten und Beobachtungen vertiefte Einsichten gewonnen werden können. Insbesondere

65 Dieses ist weithin anderer Art als das der Königspsalmen (vgl. etwa Ps 2) und später in Psalterteilbuch IV und V Gezeichnete, welches den Psalter in der Tendenz (proleptisch) mit der Chronik verbindet (vgl. Ballhorn, „David“, S. 23f.). Zum Gesagten fügt sich, dass - mit Ausnahme der Hinweise im Präskript zu Ps 60 (vgl. 1 Chr 18f.) - alle anderen biographischen David-Angaben ausschliesslich auf in den Samuel-, nicht aber den Chronikbüchern geschilderte Gegebenheiten verweisen. 
sind - stärker als es hier möglich war - die Psalmkorpora einzubeziehen. Ferner sind die Unterschiede zwischen erster und zweiter David-Sammlung mit Einsichten der Erforschung der Psaltergenese abzugleichen. ${ }^{66}$

\section{Bibliographie}

Ballhorn, E., „,Um deines Knechtes David willen' (Ps 132,10). Die Gestalt Davids im Psalter", $B N 76$ (1995), S. 16-31.

— Zum Telos des Psalters. Der Textzusammenhang des Vierten und Fünften Psalmenbuches (Ps 90-150) (в вв 139; Berlin, 2004).

Barbiero, G., Das erste Psalmenbuch als Einheit. Eine synchrone Analyse von Psalm 1-41 (ÖвS 16; Frankfurt a.M., 1999).

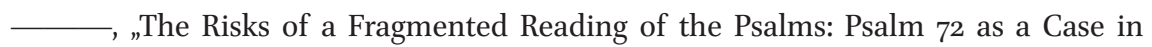
Point", ZAW 120 (2008), S. 67-91.

Bayer, B., „The Titles of the Psalms. A Renewed Investigation of an Old Problem“, Yuval 4 (1982), S. 29-123.

Botha, P.J. / Weber, B., , ,Killing Them Softly with this Song ... 'The Literary Structure of Psalm 3 and Its Psalmic and Davidic Contexts. Part I: An Intratextual Interpretation of Psalm 3“, OTE 21 (2008), S. 18-37.

—, , Killing Them Softly with this Song ... 'The Literary Structure of Psalm 3 and Its Psalmic and Davidic Contexts. Part II: A Contextual and Intertextual Interpretation of Psalm 3“, OTE 21 (2008), S. 273-297.

Buysch, C., Der letzte Davidpsalter. Interpretation, Komposition und Funktion der Psalmengruppe Ps 138-145 (sв в 63; Stuttgart, 2009).

Childs, B.S., „Psalm Titles and Midrashic Exegesis“, JSSt 16 (1971), S. 137-150.

Goulder, M., The Prayers of David (Psalm 51-72). Studies in the Psalter, II (Jsот.S 102; Sheffield, 1990).

Hartenstein, F., „,Schaffe mir Recht, JHWH!' (Psalm 7,9). Zum theologischen Profil der Teilkomposition Psalm 3-14", in: E. Zenger (ed.), The Composition of the Book of Psalms (BEThL 238; Leuven, 2010), S. 229-258.

Hossfeld, F.-L. / Zenger, E., Psalmen 51-10o (HThKAT; Freiburg i.Br., 2000).

Janowski, B., ,, Die Hindin der Morgenröte (Ps 22,1). Ein Beitrag zum Verständnis der Psalmenüberschriften“, in: F. Hartenstein / T. Willi (Hg.), Psalmen und Chronik / Psalms and Chronicles (FAT; Tübingen, 2014), im Druck.

66 Mit Rösel, Redaktion, S. 82-91, ist zu vermuten, dass die zweite David-Sammlung (innerhalb des „Elohistischen Psalters“ Ps 42-83) älter ist als die erste, der Psalter also gleichsam von seiner Mitte nach aussen gewachsen ist (wobei zunächst der Bestand von Ps 2-89 erreicht wurde und hernach Ps 9o-15o dazu kam). 
Jenni, E., Die hebräischen Präpositionen. Band 3: Die Präposition Lamed (Stuttgart, 2000).

Johnson, V.L., David in Distress. His Portrait through the Historical Psalms (Lнвотs 505; New York, NY, 2009).

Kleer, M., »Der liebliche Sänger der Psalmen Israels«. Untersuchungen zu David als Dichter und Beter der Psalmen (в в в 108; Bodenheim, 1996).

Krawczack, P., „Es gibt einen Gott, der Richter ist auf Erden!“(Ps 58,12b). Ein exegetischer Beitrag zum Verständnis von Psalm 58 (ВВВ 132; Berlin, 2001).

Millard, M., Die Komposition des Psalters. Ein formgeschichtlicher Ansatz (FAT 9; Tübingen, 1994).

Renz, J., Die althebräischen Inschriften. Teil 1: Text und Kommentar (Darmstadt, 1995).

Rösel, C., Die messianische Redaktion des Psalters. Studien zu Entstehung und Theologie der Sammlung Psalm 2-89*(CThM.BW 19; Stuttgart, 1999).

Slomovic, E., „Toward an Understanding of the Formation of Historical Titles in the Book of Psalms“, ZAW 91 (1979), S. 350-380.

Süssenbach, C., Der elohistische Psalter. Untersuchungen zu Komposition und Theologie von Ps 42-83 (FAT II/7; Tübingen, 2005).

Weber, B., Werkbuch Psalmen I. Die Psalmen 1 bis 72 (Stuttgart, 2001).

-, „Der Beitrag von Psalm 1 zu einer ,Theologie der Schrift“ “, JETh 20 (2006), S. $83-113$.

—, „,HERR, wie viele sind geworden meine Bedränger...'(Ps 3,2a). Psalm 1-3 als Ouvertüre des Psalters unter besonderer Berücksichtigung von Psalm 3 und seinem Präskript“, in: E. Ballhorn / G. Steins (Hg.), Der Bibelkanon in der Bibelauslegung. Methodenreflexionen und Beispielexegesen (Stuttgart, 2007), S. 231-251.

—, „Psalm 1 als Tor zur Tora JHwhs. Wie Ps 1 (und Ps 2) den Psalter an den Pentateuch anschliesst“, SJOT 21 (2007), S. 179-200.

_ , „Psalm 78 als ,Mitte des Psalters? - ein Versuch“, Bib. 88 (2007), S. 305-325.

, „Die Buchouvertüre Psalm 1-3 und ihre Bedeutung für das Verständnis des Psalters“, OTE 23 (2010), S. 834-845.

—, „Von der Psaltergenese zur Psaltertheologie: der nächste Schritt der Psalterexegese?! Einige grundsätzliche Überlegungen zum Psalter als Buch und Kanonteil“, in: E. Zenger (ed.), The Composition of the Book of Psalms (BEThL 238; Leuven, 2010), S. 733-744.

- Werkbuch Psalmen III. Theologie und Spiritualität des Psalters und seiner Psalmen (Stuttgart, 2010).

—, „Das königlich-davidische Danklied 2 Sam 22 / Psalm 18 im Kontext von Psalm 1-18. Eine (proto)kanonische Lesung von Ende der Samuelbücher her zum Anfangsbereich des Psalters hin“, in: S. Attard / M. Pavan (eds.), "Canterò in eterno le misericordie del Signore" (Sal 89,2) (AnBib; Roma, 2014), im Druck.

Wilson, G.H., The Editing of the Hebrew Psalter (SBL.DS 76; Chico, CA, 1985). 